\title{
Growth regulation in tip-growing cells that develop on the epidermis
}

\author{
Suvi Honkanen and Liam Dolan
}

\section{Address}

Department of Plant Sciences, University of Oxford, OX1 3RB, UK

Corresponding author: Dolan, Liam (liam.dolan@plants.ox.ac.uk)

\begin{abstract}
Summary
Plants develop tip-growing extensions - root hairs and rhizoids - that initiate as swellings on the outer surface of individual epidermal cells. A conserved genetic mechanism controls the earliest stages in the initiation of these swellings. The same mechanism controls the formation of multicellular structures that develop from swellings on epidermal cells in early diverging land plants. Details of the molecular events that regulate the positioning of the swellings involve sterols and phosphatidylinositol phosphates. The final length of root hairs is determined by the intensity of a pulse of transcription factor synthesis. Genes encoding similar transcription factors control root hair development in cereals and are potential targets for crop improvement.
\end{abstract}

\section{Introduction}

Tip growth is a mechanism of cell expansion in which growth is restricted to a small area at the apex of the elongating cell (Figure 1) [1]. Virtually all land plants develop tip-growing cells, which may have roles in reproduction (pollen tubes), or function in nutrient uptake and anchorage (root hairs and rhizoids). Here we review recent discoveries, which reveal that an ancient mechanism controls the emergence of tipgrowing cells from single epidermal cells, shed light on the molecular mechanisms that define the site of root hair outgrowth and demonstrate how the size of tipgrowing root hairs is determined.

\section{A conserved mechanism controls the initiation of epidermal cell outgrowth from the plane of the epidermis in land plants}

Root hairs protrude from cells in the outermost layer of the root - the epidermis. Root hair development begins with outward swelling of the cell wall on the outer surface of the epidermis as a result of the activity of cell wall modifying enzymes, such as expansins [1-3]. At first the protuberance expands relatively slowly, but after the tip growth apparatus is in place, the growth rate accelerates to $1-2 \mu \mathrm{m}$. $\mathrm{min}^{-1}$ [4]. 
A group of basic helix-loop-helix transcription factors - the class I ROOT HAIR DEFECTIVE SIX-LIKE (RSL) proteins - control the earliest stages of root hair development where the epidermal cell wall expands out of the surface plane. In Arabidopsis, two closely related class I RSL family genes RHD6 and RSL1 are expressed specifically in root epidermal cells that will develop root hairs, and mutants defective in RHD6 and RSL1 function are roothairless (Figure 2) [5-7]. Class I $R S L$ genes were also recently shown to control root hair development in grasses, where they are sufficient for the development of root hairs when expressed in root epidermal cells. Overexpression of class I RSL genes in rice (Oryza sativa) or the grass model species Brachypodium distachyon resulted in the development of root hairs in every root epidermal cell $[8,9]$. These findings indicate that the angiosperm class I $R S L$ genes are required for root hair cell differentiation and root hair outgrowth.

What was the ancestral role of the class I RSL genes? Microfossil evidence suggests that liverworts are likely the most ancestral lineage of land plants that diverged from the lineage leading to vascular plants soon after the first plants colonised land around 470 million years ago $[10,11]$. However, the relative phylogenetic positions of the early diverging groups of extant land plants - liverworts, mosses and hornworts - remain largely unresolved in recent molecular phylogenies. Therefore, characterising class I RSL gene function in more than one of these taxa can provide more reliable information on the ancestral role of the class I RSL genes.

The single copy class I RSL gene MpRSL1 in the liverwort Marchantia polymorpha is required for the formation of filamentous rooting cells called rhizoids, which are morphologically similar to root hairs of vascular plants and form the entire rooting system in haploid liverwort gametophytes [6]. Similarly, the two class I RSL genes PpRSL1 and PpRSL2 in the moss Physcomitrella patens are required for rhizoid development [7]. In addition, the M. polymorpha RSL1 gene is required for the development of a range of multicellular structures derived from single epidermal cells that bulge outward breaking the plane of the epidermis [6]. These structures include mucilage papillae, reproductive propagules called gemmae and multicellular slime papillae. Likewise, the development of bicellular papillae derived from single epidermal cells in P. patens is defective in the Pprs/1 Pprs/2 mutants. These findings indicate that class I RSL genes of liverworts and mosses control the development of both unicellular and multicellular structures that develop from single epidermal cells. Together, these data suggest that class I RSL genes are likely to have functioned as positive regulators of the early stage of epidermal cell outgrowth during the morphogenesis of both unicellular and multicellular structures in the last common ancestor of land plants.

\section{Evolution of antagonistic regulators of root hair elongation}

LOTUS JAPONICUS ROOTHAIRLESS1-LIKE (LRL) family genes encode for basic helixloop-helix transcription factors that are required for root hair development in the angiosperms Lotus japonicus and $A$. thaliana $[12,13]$. However, unlike class I RSL genes, they are also involved in other aspects of development and are expressed 
throughout the plant $[13,14]$. The liverwort $M$. polymorpha has a single $L R L$ gene $M p L R L$, which positively regulates plant growth and rhizoid development [14]. Likewise, $P$. patens mutants that lack the function of the two $L R L$ genes (PpLRL1 and $P p L R L 2$ ) are stunted and do not develop rhizoids [15]. These data indicate that $L R L$ genes function as general growth promoters and positive regulators of rhizoid development in early diverging land plants. $A$. thaliana has six $L R L$ genes that belong to two subfamilies [14]. $L R L$ genes from subfamily I are required for root hair elongation. In contrast, $L R L$ subfamily II genes repress root hair development; plants overexpressing members of subfamily II develop short root hairs. Together these data suggest that an ancestral $L R L$ gene promoted growth, and that during the course of land plant evolution, gene duplication led to the evolution of $L R L$ subfamily I genes that promote root hair growth, and subfamily II genes that repress growth. The precise mechanism by which the genes in $L R L$ subfamilies I and II respectively promote and repress growth remains to be defined.

\section{Phosphatidylinositol phosphates and sterols mark the site of root hair outgrowth}

The formation of the RSL gene-regulated cell surface outgrowth is accompanied by a number of changes that have recently been characterised in detail in $A$. thaliana root hair development. The plant hormone auxin is involved in positioning the site root hair outgrowth on the hair forming epidermal cells (trichoblasts). In wild type $A$. thaliana, auxin synthesised at the root tip is transported through the non-hair forming epidermal cell files towards the shoot by auxin transporter proteins, resulting in a tip-high auxin gradient along the root axis $[16,17]$. The non-hair forming cell files supply auxin to the adjacent root hair forming cell files, wherein the root hair initiation sites become established at the rootward end of each cell, towards the auxin maxima at the root tip $[5,16,18,19]$. Loss-of-function mutations in the auxin influx carrier AUXIN RESISTANT1 (AUX1) result in more shootward root hair initiation $[18,19]$. Similarly, gain-of-function mutation that disrupts auxin dependent degradation of the Aux/IAA transcriptional repressor protein AUXIN RESISTANT2 (AXR2) results in more shootward root hair initiation $[5,20,21]$. These results indicate that both auxin transport and auxin responsive gene expression are required for correct positioning of root hair initiation site on root epidermal cells. Furthermore, mutants for the class I RSL gene rhd6 develop few root hairs that are also shifted shootward [5]. The root hair number and positioning defects in the rhd 6 mutants can be rescued by auxin treatment [5]. These findings indicate that auxin controls positioning the site of root hair outgrowth downstream of class I RSL genes.

Before root hair initiation, proteins required for positioning of outgrowth site accumulate in the plasma membrane at the future site of outgrowth.

Phosphatidylinositol-4-phosphate 5-kinase 3 (PIP5K3) and RHO-RELATED PROTEIN FROM PLANTS2 (ROP2) GTPases are required for outgrowth site determination as their over-expression leads to initiation of multiple hairs from a single hair forming epidermal cell [22-24]. Furthermore, ROP GTPases are required for focusing cell expansion at the apex of elongating root hairs: plants expressing constitutively active ROP2 or ROP6 develop swollen depolarised root hairs [24,25]. Stanislas et al., 2015 
[26] demonstrated that ROP2, ROP6, PIP5K3 as well as DYNAMIN RELATED PROTEIN (DRP) $1 A$ and DRP2B involved in Clathrin-mediated endocytic vesicle trafficking $[27,28]$ co-localise in a distinct sterol-enriched domain at the future root hair outgrowth site. Furthermore, at the onset of outgrowth, the AGC VIII kinase D6 PROTEIN KINASE (D6PK) was recruited into this sterol-enriched membrane domain in sterol- and phosphatidylinositol-4,5-bisphosphate- $\left(\operatorname{Ptdlns}(4,5) \mathrm{P}_{2}\right)$ dependent manner (Figure 2) [26]. Therefore, while the role of D6PK in root hair development has not yet been established, these findings suggest that sterols and phosphatidylinositol phosphates contribute to the recruitment of proteins to a distinct plasma membrane domain focused at the future site of root hair initiation.

\section{Arabidopsis class II RSL gene $R S L 4$ modulates root hair length}

After root hair tip growth has been initiated from the swelling on the outer surface of the epidermal cell, the root hair begins to elongate rapidly. In Arabidopsis, the duration of the tip growth phase, and therefore the final length of root hairs, is defined by the levels of the class II RSL basic helix-loop-helix transcription factor ROOT HAIR DEFECTIVE SIX-LIKE4 (RSL4) $[29,30]$. Class I RSL genes (RHD6 and RSL1) positively regulate the transcription of $R S L 4$ mRNA, which accumulates in a pulse-like fashion in root hair forming cells at the root elongation zone (Figure 2) [29,30]. RSL4GFP fusion protein can first be observed 2 hours before the emergence of root hair outgrowth. RSL4-GFP protein level peaks 4 hours later, and then gradually declines [30]. Root hair elongation continues as long as RSL4 protein persists in the nuclei of the hair cells. The RSL4 protein has a D-box motif characteristic of proteins that become degraded by the $26 \mathrm{~S}$ proteasome. Introducing a mutation that disrupts the RSL4 D-box motif increased the longevity of the protein, resulting in extended tip growth phase and longer root hairs [30]. Together, these results indicate that the length of Arabidopsis root hairs is defined by the duration of the presence of RSL4 protein in the nuclei of hair cells.

Root hairs have been shown to be important for uptake of phosphate, which is often the limiting nutrient for plant growth [31-34]. Phosphorus ions are negatively charged and bind tightly to positively charged clay particles in soil, and are thus relatively unavailable to plants. Plants have evolved many physiological, morphological and biochemical adaptations in order to increase phosphorus uptake under low phosphate conditions (Reviewed in [35]). One of these adaptations is the regulation of root hair length [36,37]. Increase in root hair length in low phosphate conditions results in increased surface area of the rooting system and exploration of larger volume of the soil, which has been shown to be associated with increased phosphate uptake and higher biomass [31-34,38].

The increase in Arabidopsis root hair elongation in response to phosphate starvation is exerted through RSL4 [29]. Datta et al., 2015 [30] discovered that a greater pulse of RSL4 protein synthesis occurs in plants grown in low phosphate medium than in replete phosphate. This increase of RSL4 protein synthesis during root hair initiation resulted in longer persistence of the RSL4 protein and longer root hairs [30]. Low 
phosphate can induce the development of root hairs in the rhd $6 \mathrm{rs} / 1$ double mutant background, but not in the $r h d 6$ rs/1 rs/4 triple mutant background, indicating that the function of $R S L 4$ is required for the low phosphate response of the root hairs [29]. Therefore, RSL4 integrates endogenous signals transmitted by the class I RSL genes, and environmental factors such as low phosphate to modulate root hair length.

Both auxin and RSL4 positively regulate root hair elongation in A. thaliana. The expression of RSL4 is increased in roots treated with artificial auxin, indicating that at least part of auxin-regulated root hair elongation is dependent on RSL4 activity [29]. To elucidate how RSL4 promotes root hair elongation, Vijayakumar et al., 2016 [39] identified immediate downstream targets of RSL4. 34 putative $R S L 4$ downstream target genes were identified based on their increased expression after induction of RSL4 activity in the presence of translational inhibitor. The putative RSL4 downstream targets include genes that function in cell wall synthesis, membrane trafficking and cell signalling [39]. These genes are likely effectors of growth. Taken together, these data indicate that $R S L 4$ is a key regulator of root hair growth that integrates a variety of signals, such as low phosphate and auxin - and acts down stream of the class I RSL genes.

\section{Root hair length in cereal crops is regulated by $R S L$ genes}

Longer root hairs allow the plant to explore and extract nutrients from a greater volume of soil. Therefore, engineering plants with longer root hairs may produce more nutrient uptake efficient crop varieties [34]. Class I and class II RSL genes are present in the genomes of cereal grasses $[8,9,40]$. Class I $R S L$ genes in rice $(0$. sativa) and the cereal model species $B$. distacyon are required for root hair differentiation. Furthermore, expression of class I RSL1 proteins in root epidermal cells is sufficient for root hair development in cereals; $O$. sativa or $B$. distachyon plants expressing class I RSL genes behind the strong constitutive rice ubiquitin promoter develop root hairs on every root epidermal cell $[8,9]$.These data demonstrate that $R S L$ genes control root hair development in cereal grasses.

Recently, a pioneering study demonstrated how class II RSL genes play an important role in determining root hair length and cereal growth in low nutrient soils [40]. Bread wheat (Triticum aestivum) is an allohexaploid, with three subgenomes (AABBDD) that originated from two separate polyploidisation events between three diploid wild grass species. The first polyploidisation event produced allotetraploid durum wheat (AABB), which further hybridised with diploid goatgrass giving rise to allohexaploid bread wheat (AABBDD), the major wheat variety grown today [41]. Each of the three bread wheat subgenomes contains a copy of TaRSL4, a wheat homolog of the $A$. thaliana class II RSL gene RSL4. Therefore, each of these polyploidisation events was associated with an increase in TaRSL4 gene copy number. Han et al., 2015 [40] observed root hairs in natural and synthetic allotetraploid and allohexaploid wheat and their diploid progenitors to estimate the impact of the polyploidisation events that took place during wheat domestication on 
root hair length. Root hairs of allotetraploid wheat (AABB) were significantly longer than those of their diploid ancestors [40]. These longer root hairs were correlated with higher steady-state TaRSL4 mRNA levels, which is consistent with the hypothesis that RSL4 proteins control root hair length in cereals. Furthermore, overexpression of TaRSL4 in an elite bread wheat cultivar resulted in longer root hairs and enhanced shoot biomass in plants grown under low nutrient conditions [40]. Therefore, the polyploidisation event that produced durum wheat (AABB) and the associated increase in class II $R S L$ gene copy number is likely to have resulted in elevated class II RSL gene expression and therefore longer root hairs. It can be hypothesised that longer root hairs contributed to the suite of traits that increased vigor of the polyploid durum wheat relative to its diploid ancestors. Furthermore, these findings suggest that the role of class II RSL genes as modulators of root hair length is conserved between dicots and monocot cereal crops.

\section{Conclusions}

Recent discoveries have demonstrated how an ancient mechanism involving the RSL family of basic helix-loop-helix transcription factors control the formation of the polar outgrowth from epidermal cells at the plant surface. Research in $A$. thaliana has identified a suite of molecular events that occur at the site of outgrowth. Discovering the mechanistic the link between these two processes and how regulatory mechanisms evolved during plant evolution will be a priority.

\section{Acknowledgements}

We are grateful to Dr. Chulmin Kim for provision of the rice root hair image. This work was supported by European Research Council Advanced Grant (EVO500; Project No: 25028) to LD.

\section{References}

1. Rounds CM, Bezanilla M: Growth Mechanisms in Tip-Growing Plant Cells. Annu Rev Plant Biol 2013, 64:243-265.

* Comprehensive review of the cell growth mechanisms that operate during tip growth in plant cells.

2. Ma N, Wang Y, Qiu S, Kang Z, Che S, Wang G, Huang J: Overexpression of OsEXPA8, a root-specific gene, improves rice growth and root system architecture by facilitating cell extension. PLOS ONE 2013, 8:e75997.

3. Baluška F, Salaj J, Mathur J, Braun M, Jasper F, Šamaj J, Chua N-H, Barlow PW, Volkmann D: Root hair formation: F-actin-dependent tip growth is initiated by local assembly of profilin-supported F-actin meshworks accumulated 
within expansin-enriched bulges. Dev Biol 2000, 227:618-632.

4. Dolan L, Duckett CM, Grierson C, Linstead P, Schneider K, Lawson E, Dean C, Poethig $\mathrm{S}$, Roberts $\mathrm{K}$ : Clonal relationships and cell patterning in the root epidermis of Arabidopsis. Development 1994, 120:2465-2474.

5. Masucci JD, Schiefelbein JW: The rhd6 mutation of Arabidopsis thaliana alters root-hair initiation through an auxin- and ethylene-associated process. Plant Physiol 1994, 106:1335-1346.

6. Proust $\mathrm{H}$, Honkanen $\mathrm{S}$, Jones VAS, Morieri $\mathrm{G}$, Prescott $\mathrm{H}$, Kelly $\mathrm{S}$, Ishizaki $\mathrm{K}$, Kohchi T, Dolan L: RSL class I genes controlled the development of epidermal structures in the common ancestor of land plants. Curr Biol 2016, 26:93-99.

** RSL class I genes promote the development of unicellular tip-growing projections (rhizoids) and multicellular structures (papillae and gemmae) that develop from single epidermal cells in the liverwort $M$. polymorpha and moss $P$. patens. This suggests that the ancestral function of $R S L$ class I genes was to promote the early outgrowth of individual epidermal cells which may then pursue unicellular development or multicellular development in a class I RSLindependent manner.

7. Menand B, Yi K, Jouannic S, Hoffmann L, Ryan E, Linstead P, Schaefer DG, Dolan L: An ancient mechanism controls the development of cells with a rooting function in land plants. Science 2007, 316:1477-1480.

8. Kim CM, Dolan L: RSL class I gene function is conserved among angiosperms and expression pattern defines root hair cell pattern in grasses. PLOS Genet 2016 in press.

9. Kim CM, Han C, Dolan L: $R S L$ class I genes positively regulate root hair development in Oryza sativa. New Phytol 2016 In press.

10. Steemans $\mathrm{P}$, Herisse AL, Melvin J, Miller MA, Paris F, Verniers J, Wellman CH: Origin and radiation of the earliest vascular land plants. Science 2009, 324:353-353.

11. Wellman $\mathrm{CH}$, Gray J: The microfossil record of early land plants. Philos Trans $R$ Soc Lond B 2000, 355:717-732.

12. Karas B, Murray J, Gorzelak M, Smith A, Sato S, Tabata S, Szczyglowski K: Invasion of Lotus japonicus root hairless 1 by Mesorhizobium loti involves the nodulation factor-dependent induction of root hairs. Plant Physiol 2005, 137:1331-1344.

13. Karas B, Amyot L, Johansen C, Sato S, Tabata S, Kawaguchi M, Szczyglowski K: Conservation of lotus and Arabidopsis Basic Helix-Loop-Helix proteins reveals new players in root hair development. Plant Physiol 2009, 151:11751185. 
14. Breuninger $H$, Thamm A, Streubel $S$, Sakayama $H$, Nishiyama T, Dolan L: Diversification of a transcription factor family led to the evolution of antagonistically acting genetic regulators of root hair growth. Curr Biol 2016, 26:1622-1628.

**LRL genes in early diverging land plants promote growth. Gene duplication during the course of land plant evolution led to the evolution of two $L R L$ subfamilies with antagonistic functions; one promotes growth and the other represses growth.

15. Tam THY, Catarino B, Dolan L: Conserved regulatory mechanism controls the development of cells with rooting functions in land plants. Proc Natl Acad Sci USA 2015, doi:10.1073/pnas.1416324112.

16. Jones AR, Kramer EM, Knox K, Swarup R, Bennett MJ, Lazarus CM, Leyser HMO, Grierson CS: Auxin transport through non-hair cells sustains root-hair development. Nat Cell Biol 2008, 11:78-84.

17. Sabatini S, Beis D, Wolkenfelt H, Murfett J, Guilfoyle T, Malamy J, Benfey P, Leyser $\mathrm{O}$, Bechtold N, Weisbeek $\mathrm{P}$, et al.: An auxin-dependent distal organizer of pattern and polarity in the Arabidopsis root. Cell 1999, 99:463-472.

18. Grebe M, Friml J, Swarup R, Ljung K, Sandberg G, Terlou M, Palme K, Bennett MJ, Scheres B: Cell polarity signaling in Arabidopsis involves a BFA-sensitive auxin influx pathway. Curr Biol 2002, 12:329-334.

19. Ikeda Y, Men S, Fischer U, Stepanova AN, Alonso JM, Ljung K, Grebe M: Local auxin biosynthesis modulates gradient-directed planar polarity in Arabidopsis. Nat Cell Biol 2009, 11:731-738.

20. Nagpal P, Walker LM, Young JC, Sonawala A, Timpte C, Estelle M, Reed JW: AXR2 encodes a member of the Aux/IAA protein family. Plant Physiol 2000, 123:563-574.

21. Wilson AK, Pickett FB, Turner JC, Estelle M: A dominant mutation in Arabidopsis confers resistance to auxin, ethylene and abscisic acid. Mol Gen Genet 1990, 222:377-383.

22. Kusano H, Testerink C, Vermeer JEM, Tsuge T, Shimada H, Oka A, Munnik T, Aoyama T: The Arabidopsis phosphatidylinositol phosphate 5-kinase PIP5K3 Is a key regulator of root hair tip growth. Plant Cell 2008, 20:367-380.

23. Stenzel I, Ischebeck T, Konig S, Holubowska A, Sporysz M, Hause B, Heilmann I: The type $B$ phosphatidylinositol-4-phosphate 5-kinase 3 is essential for root hair formation in Arabidopsis thaliana. Plant Cell 2008, 20:124-141.

24. Jones MA, Shen JJ, Fu Y, Li H, Yang Z, Grierson GS: The Arabidopsis Rop2 GTPase Is a positive regulator of both root hair initiation and tip growth. Plant Cell 2002, 14:763-776. 
25. Molendijk AJ, Bischoff F, Rajendrakumar CS, Friml J, Braun M, Gilroy S, Palme $\mathrm{K}$ : Arabidopsis thaliana Rop GTPases are localized to tips of root hairs and control polar growth. EMBO J 2001, 20:2779-2788.

26. Stanislas T, Hüser A, Barbosa ICR, Kiefer CS, Brackmann K, Pietra S, Gustavsson A, Zourelidou M, Schwechheimer C, Grebe M: Arabidopsis D6PK is a lipid domain-dependent mediator of root epidermal planar polarity. Nat Plants 2015, 1:15162.

*The authors demonstrate that prior to root hair outgrowth the AGC VIII kinase D6 PROTEIN KINASE (D6PK) was recruited into a sterol-enriched membrane domain at the future site of outgrowth in a sterol- and phosphatidylinositol-4,5-bisphosphate- $\left(\operatorname{Ptdlns}(4,5) \mathrm{P}_{2}\right)$ dependent manner.

27. Fujimoto $\mathrm{M}$, Arimura SI, Ueda T, Takanashi H, Hayashi $\mathrm{Y}$, Nakano A, Tsutsumi $\mathrm{N}$ : Arabidopsis dynamin-related proteins DRP2B and DRP1A participate together in clathrin-coated vesicle formation during endocytosis. Proc Natl Acad Sci USA 2010, 107:6094-6099.

28. Backues SK, Korasick DA, Heese A, Bednarek SY: The Arabidopsis DynaminRelated Protein2 family is essential for gametophyte development. Plant Cell 2010, 22:3218-3231.

29. Yi K, Menand B, Bell E, Dolan L: A basic helix-loop-helix transcription factor controls cell growth and size in root hairs. Nat Genet 2010, 42:264-267.

30. Datta S, Prescott H, Dolan L: Intensity of a pulse of RSL4 transcription factor synthesis determines Arabidopsis root hair cell size. Nat Plants 2015, 1:15138.

**This study shows that the length of root hairs is determined by the amount of RSL4 protein produced: longer hairs develop in cells with relatively more RSL4 protein and shorter hairs develop in cells with relatively less RSL4 protein.

31. Gahoonia TS, Care D, Nielsen NE: Root hairs and phosphorus acquisition of wheat and barley cultivars. Plant Soil 1997, 191:181-188.

32. Gahoonia TS, Nielsen NE: Phosphorus (P) uptake and growth of a root hairless barley mutant (bald root barley, brb) and wild type in low-and highP soils. Plant Cell Environ 2003, 26:1759-1766.

33. Gahoonia TS, Nielsen NE: Barley genotypes with long root hairs sustain high grain yields in low-P field. Plant Soil 2004, 262:55-62.

34. Brown LK, George TS, Dupuy LX, White PJ: A conceptual model of root hair ideotypes for future agricultural environments: what combination of traits should be targeted to cope with limited P availability? Ann Bot 2013, 112:317-330. 
35. Vance $\mathrm{CP}$, Uhde Stone $\mathrm{C}$, Allan DL: Phosphorus acquisition and use: critical adaptations by plants for securing a nonrenewable resource. New Phytol 2007, 157:423-447.

36. Bates TR, Lynch JP: Stimulation of root hair elongation in Arabidopsis thaliana by low phosphorus availability. Plant Cell Environ 1996, 19:529-538.

37. Ma Z, Bielenberg DG, Brown KM: Regulation of root hair density by phosphorus availability in Arabidopsis thaliana. Plant Cell Environ 2001, 24:459-467.

38. Bates TR, Lynch JP: Plant growth and phosphorus accumulation of wild type and two root hair mutants of Arabidopsis thaliana (Brassicaceae). Am J Bot 2000, 87:958-963.

39. Vijayakumar P, Datta S, Dolan L: ROOT HAIR DEFECTIVE SIX-LIKE4 (RSL4) promotes root hair elongation by transcriptionally regulating the expression of genes required for cell growth. New Phytol 2016, doi:10.1111/nph.14095.

40. Han Y, Xin M, Huang K, Xu Y, Liu Z, Hu Z, Yao Y, Peng H, Ni Z, Sun Q: Altered expression of TaRSL4gene by genome interplay shapes root hair length in allopolyploid wheat. New Phytol 2015, 209:721-732.

**This paper is a tour de force and demonstrates that TaRSL4 is expressed at high levels in wheat cultivars with long root hairs. This variation likely arose during the polyploidisation events that occurred during the evolution of hexaploid wheat. Overexpression of TaRSL4 in transgenic wheat increases root hair length and biomass. This suggests that regulators such as RSL4 could be useful in understanding how crop root hairs grow and may provide tools for plant breeding in the future.

41. International Wheat Genome Sequencing Consortium (IWGSC): A chromosome-based draft sequence of the hexaploid bread wheat (Triticum aestivum) genome. Science 2014, 345:1251788. 